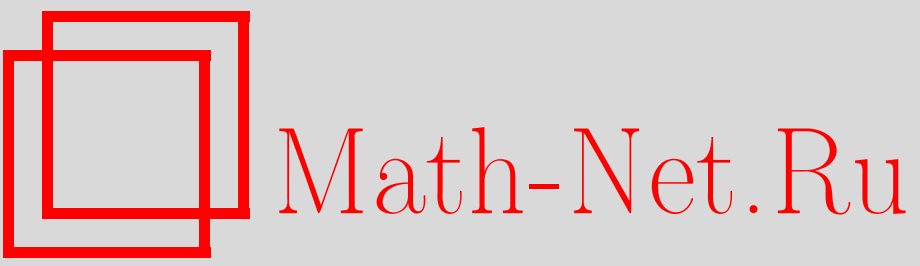

А. А. Болибрух, Регулярные особые точки как изомонодромные слияния фуксовых, УМН, 2001, том 56, выпуск 4, 135-136

DOI: https://doi.org/10.4213/rm420

Использование Общероссийского математического портала Math-Net.Ru подразумевает, что вы прочитали и согласны с пользовательским соглашением

http://www . mathnet.ru/rus/agreement

Параметры загрузки:

IP: 54.210 .77 .194

26 апреля 2023 г., 13:02:37 


\title{
РЕГУЛЯРНЫЕ ОСОБЫЕ ТОЧКИ КАК ИЗОМОНОДРОМНЫЕ СЛИЯНИЯ ФУКСОВЫХ
}

\author{
А. А. Болиьрух
}

Рассмотрим изомонодромное семейство фуксовых систем $p$ линейных уравнений

$$
\frac{d y}{d z}=\left(\sum_{i=1}^{n} \frac{B_{i}(a)}{z-a_{i}}\right) y
$$

на сфере Римана $\mathbb{P}^{1}$, где матрицы $B_{i}(a)$ аналитически зависят от положения набора особых точек $a=\left(a_{1}, \ldots, a_{n}\right)$. Изомонодромность семейства (1) означает существование такой фундаментальной матрицы $Y(z, a)$ семейства, которая аналитична по аргументам $z, a$ и которая при любом фиксированном значении $а$ имеет одну и ту же монодромию (см. подробнее [1]). (Естественная область определения семейства (1) - универсальная накрьвающая сферы Римана с выколотыми особыми точками, но поскольку нас будут интересовать лишь малые перемещения особых точек по отношению к некоторому их начальному положению, то можно считать, что все происходит на одном листе накрытия.)

Будем говорить, что система уравнений

$$
\frac{d y}{d z}=B(z) y
$$

с особыми точками $b_{1}, \ldots, b_{k}$ является резултатом изомонодромного слияния особых точек семейства (1), если матрица $\sum_{i=1}^{n} \frac{B_{i}(a)}{z-a_{i}}$ стремится к $B(z)$, когда для любого $i$ точки $a_{i_{1}}, \ldots, a_{i_{m_{i}}}$ стремятся к точке $b_{i}$, оставаясь каждая в своем секторе с вершиной в этой точке.

Будем называть такое слияние нормализованным, если при этом слиянии изомонодромная матрица семейства (1) стремится к некоторой фундаменталњной матрице итоговой системы (2).

В работе [1] доказано, что нормализованное изомонодромное слияние фуксовых особых точек приводит к появлению лишь регулярных особых точек, т.е. таких точек, в которых решения итоговой системы (2) имеют не более чем степенной рост. Основным результатом настоящей работы является следующее утверждение.

Теорема. Любая система (2) линейных дифференциальных уравнений с регулярными особыми точками на сфере Римана является результатом нормализованного изомонодромного слияния особых точек семейства фуксовьх систем уравнений.

ДокАЗАТЕльство. Теорему достаточно доказать для случая, когда все особые точки системы (2) фуксовы за исключением одной. Можно считать, что эта точка расположена в нуле и что матрица коэффициентов системы имеет в окрестности этой точки следующий вид: $B(z)=$ $\frac{B_{0}(z)}{z^{r+1}}$, где $B_{0}(z)$ голоморфна в нуле и не зануляется там, а целое число $r$, назьваемое рангом Пуанкаре системы (2), положительно.

Для фундаментальной матрицы $Y(z)$ системы (2), построенной по специальному, так называемому Левелевскому, базису пространства решений в окрестности точки нуль, имеет место следующее тождество (см. [2]): $Y(z)=U(z) z^{A} z^{E}$, где $A$ - целочисленная диагональная матрица нормирований базиса, $E$ - постоянная матрица и матричная функция $U(z)$ голоморфна в нуле.

Покажем, что матрицу $U(z)$ можно представить в следующем виде:

$$
U(z)=\Gamma(z) V(z), \quad \Gamma(z)=\Gamma_{0} z^{L_{1}} \Gamma_{1} z^{-L_{1}} \ldots z^{L_{s}} \Gamma_{s} z^{-L_{s}} z^{K},
$$

Работа выполнена при поддержке Российского фонда фундаментальных исследований (грант № 99-01-00157). 
где все $\Gamma_{i}$ - постоянные матрицы, $L_{j}=K_{1}+\cdots+K_{j}$, все матрицы $K_{i}, K$ и матрица $K-L_{s}$ являются диагональньми целочисленньми матрицами с неотрицательными элементами, $V(z)$ голоморфно обратима в нуле и при всех $j$ матричные функции $z^{L_{j}} \Gamma_{j} z^{-L_{j}}$ голоморфны в точке бесконечность.

Умножив $U(z)$ слева на неособую постоянную матрицу $S$, переставим ее строки так, чтобы первые $l$ строк матрицы $U(0)$, имеющей ранг $l$, были линейно независимыми. Обозначим матрицу $S U$ вновь через $U$. Найдется такая невырожденная матрица $T$, первые $l$ строк которой совпадают с единичной матрицей, что у матрицы $T U(0)$ последние $p-l$ строк нулевые. Поэтому $T U(z)=$ $z^{L_{1}} U^{\prime}(z)$ с голоморфиой матричной функцией $U^{\prime}(z)$. Обозначим $\Gamma_{0}=S^{-1} T^{-1}$.

Будем считать, что диагональные элементы матрицы $L_{1}$ образуют невозрастающую последовательность (доказательство в общем случае легкой модификацией сводится к рассматриваемому). Пусть первые $m$ строк матрицы $U^{\prime}(0)$ линейно независимы, а $(m+1)$-я строка является их линейной комбинацией. Найдется такая невырожденная матрица $Q$, первые $m$ строк которой совпадают с единичной матрицей, что у матрицы $Q U^{\prime}(0)$ строка с номером $(m+1)$ - нулевая. Тогда $Q U^{\prime}(z)=z^{K_{2}} U^{\prime \prime}(z)$ с голоморфной матрицей $U^{\prime \prime}(z)$. Обозначим $\Gamma_{1}=Q^{-1}$. По построению матрица $z^{L_{1}} \Gamma_{1} z^{-L_{1}}$ голоморфнна в бесконечности и $U(z)=\Gamma_{0} z^{L_{1}} \Gamma_{1} z^{-L_{1}} z^{L_{1}+K_{2}} U^{\prime \prime}(z)$.

Обозначим матрицу $L_{1}+K_{2}$ через $L_{2}$. В предположении, что элементы матрицы $L_{2}$ образуют невозрастающую последовательность, рассмотрим первые $k$ линейно независимых строк матрицы $U^{\prime \prime}(0)$ и т. д. После конечного числа шагов получим тождество (3).

Рассмотрим матричную функцию $H(z, a)=\Gamma_{0} H_{1}(z, a) \cdots H_{s}(z, a) G(z, a)$, где $H_{i}(z, a)=$ $F_{i} \Gamma_{i} D_{i}$ и где, в свою очередь, $F_{i}=\prod_{j=1}^{l_{i}}\left(z-a_{j}^{i}\right)^{N_{j}^{i}}, D_{i}=\prod_{j=1}^{l_{i}}\left(z-c_{j}^{i}\right)^{-N_{j}^{i}}, G(z, a)=$ $\prod_{j=1}^{l}\left(z-a_{j}^{s+1}\right)^{N_{j}^{s+1}}$, все элементы матриц $N_{j}^{i}$ равны нулю кроме одного диагонального элемента, равного единице, $l_{i}=\operatorname{tr} L_{i}, \sum_{j=1}^{l_{i}} N_{j}^{i}=L_{i}, \sum_{j=1}^{l} N_{j}^{s+1}=K$ и все точки $a_{j}^{i}, c_{j}^{i}, b_{l}$ различны.

Из построения следует, что функция $H(z, a)(\Gamma(z))^{-1}$ голоморфна в бесконечности, семейство систем уравнений с фундаментальной матрицей $Y(z, a)=H(z, a)(\Gamma(z))^{-1} Y(z)$ изомонодромно, фуксово при всех $a_{j}^{i}, c_{j}^{i}, b_{l}$ и матричная функция $Y(z, a)$ стремится к $Y(z)$, когда все точки $a_{j}^{i}, c_{j}^{i}$ стремятся к нулю.

Заметим, что у построенного семейства фуксовых уравнений все особые точки $a_{j}^{i}, c_{j}^{i}$ являются ложными, т.е. решения в этих точках не ветвятся. Из работы [3] следует, что $\operatorname{tr} K \leqslant r p(p-1) / 2$, поэтому число таких точек оценивается сверху числом $2 \operatorname{tr} L_{1}+\cdots+2 \operatorname{tr} L_{s}+\operatorname{tr} K$, которое не превосходит числа $(r p(p-1) / 2)^{2}$.

\section{СПИСОК ЛИТЕРАТУРЫ}

[1] А. А. Болибрух // Труды МИАН. 1998. Т. 221. С. 127-142. [2] А. А. Болибрух // Труды МИАН. 1994. Т. 206. [3] E. Corel // C. R. Acad. Sci. Paris. 1999. V. 328. P. 983-986. 